\title{
COMETARY VERSUS ASTEROIDAL ORIGIN OF CHONDRITIC METEORITES
}

\author{
GEORGE W. WETHERILL \\ University of California, Los Angeles
}

Much of what we know about the early history of the solar system has been learned from the study of meteorites. This results from the fact, demonstrated by isotopic age measurements, that all of the various classes of stone and iron meteorites were formed $4.6 \times 10^{9} \mathrm{yr}$ ago within a short period of time, probably less than 100 million yr in duration. This is also the age of Earth and the Moon and may be presumed to be the time of formation of the solid bodies in the solar system. Measurements of the products of the decay of the extinct radioactive isotopes ${ }^{129} \mathrm{Xe}$ and ${ }^{244} \mathrm{Pu}$ show, furthermore, that the formation of these solid bodies occurred within 100 million yr of the time of separation of the solar nebula from interstellar matter. Except for physical fragmentation into smaller bodies, the chemical and mineralogical composition of most meteorites has been essentially unaltered since this time during the formation interval of the solar system.

This situation contrasts with that found on Earth, where geological processes have essentially erased the record of the first 25 percent of Earth's history. The Moon is now known to be intermediate between Earth and meteorites in this regard. Although the record of the Moon's early history is preserved to a much greater extent than that of Earth, significant formation of lunar rocks occurred at least as recently as $3.3 \times 10^{9} \mathrm{yr}$ ago. Although the best preserved record of the early history of the solar system is to be found in the meteorites, these data are difficult to interpret because, unlike rocks from Earth and the Moon, we have no definite information regarding the sources in the solar system of these rocks that are now colliding with Earth. Were such information to become available, the role of meteorites would become fully equivalent to that of lunar samples in experimental studies of the origin of the solar system.

\section{SUMMARY OF EARLIER WORK}

From the work of Öpik (1951) we know that neither the meteoritic fragment nor its parent body can have been in its present Earth-crossing orbit for the entire history of the solar system. This is because these orbits are stable 
with respect to planetary impact or ejection from the solar system for no more than 100 million yr. From the cosmic-ray-exposure ages we also know that the meteorite was broken from a larger body late in the history of the solar system. These facts require us to find some place in the solar system where we can "store" the larger body from which the meteorite was fragmented for most of the solar system's history, and then we must find a way to transfer more recently either the fragment or the parent body itself from its "storage place" into an Earth-crossing orbit, from which further fragmentation and collision with Earth are possible.

The problem of identifying the source of meteorites can therefore be approached from the point of view of finding an appropriate storage place. The surfaces of a planetary body such as Mars or the Moon have been proposed, but are very unlikely to be satisfactory. It is hard to see how a fragment of meteoritic size can be accelerated to planetary escape velocities without complete destruction, or without at least experiencing shock metamorphism far exceeding that found in most meteorites.

For this reason, as well as others, smaller bodies are more promising candidates. The two principal types of smaller bodies in the solar system are the comets and the asteroids. The associated storage spaces are the cometary cloud of Oort and the asteroid belt, respectively. No other associations of small bodies and storage places are known at present; it is conceivable that in the outer solar system there are additional unobservable families of small bodies of some kind. However, it seems most fruitful to give first consideration to known classes of bodies, rather than to entirely speculative ones.

We have insufficient knowledge of the chemical or mineralogical composition, or for that matter, even the mean density of comets and asteroids to permit identification of any class of meteorites with these bodies on the basis of data of these kinds. Also, because the chemistry and mineralogy of the meteorites has been fixed since some time during the formation of the solar system, it is possible that the establishment of this chemistry and mineralogy preceded the time at which the present parent bodies were formed. Consequently, it is very difficult to make even plausible arguments concerning the kind of objects that could be derived from comets or asteroids without far more understanding than we possess regarding the processes by which these objects were formed.

It is possible that identification of meteorites with their sources must await in situ analyses and other studies by suitable spacecraft. However, prior to such studies, there is a body of dynamical evidence bearing on this problem that can prove valuable in making plausible inferences regarding this identification and that can provide reasonable hypotheses useful in planning such missions. Application of dynamical data to this problem has been described (Arnold, 1965a,b; Öpik, 1966; Wetherill, 1968a,b; Wetherill, 1969). The purpose of this report is to update this earlier work and describe the progress that has been made in the last few years. 
As discussed in these earlier reports, it may be expected that there will be a relationship between the distribution of orbits from which meteorites impact Earth and the orbit of their source. At the time the meteorite was fragmented from a larger body, its initial orbit was approximately that of the larger body, because the large unshocked fragments surviving the fragmentation event will have a low velocity in the center-of-mass reference frame. However, these initial orbits will not be identical; and, with the passage of time, they will evolve into a distribution of orbits, some of which will be Earth crossing ones from which meteorites will be derived. The approach that has been taken is to consider various source orbits or "initial orbits" and see which, if any, of these evolve into orbits distributed in such a way as to correspond to the observed data. It is also necessary that the time interval between the fragmentation event that starts the "cosmic-ray clock" and Earth impact be in agreement with cosmic-ray-exposure ages, and furthermore, that any acceptable source provide a mass yield in accord with the observed meteorite flux.

The observed data consist of the time of fall of several hundred meteorites, the apparent radiants and exposure ages of about 100 , and a very few complete orbits, only two of which can be considered well determined, Př́bram and Lost City. Only for the most abundant class of meteorites, the chondrites, are these observed data sufficiently complete to be useful. In addition, orbits have been determined for a large number of bright fireballs falling within the Prairie Network. (See McCrosky, 1967.) These data for chondrites and fireballs are shown in figures 1 through 4.

In my previous studies (Wetherill, 1968a,b; 1969), I have considered initial orbits corresponding to those of the Moon, the Earth-crossing Apollo asteroids,

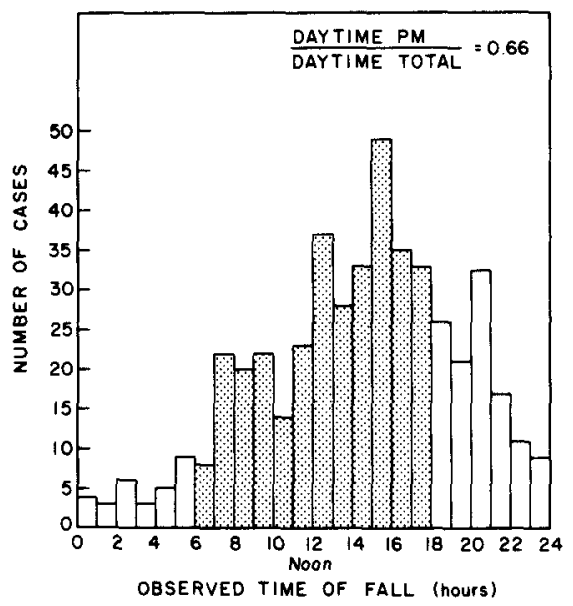

Figure 1.- Observational data for time of fall of chondritic meteorites. Dotted area indicates daytime falls, when social biases are of lesser importance. 


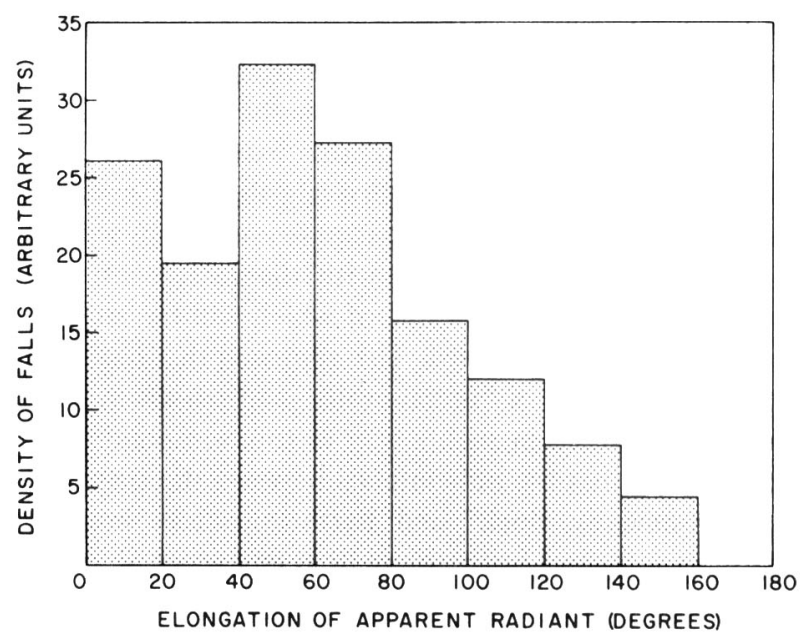

Figure 2.-Observed distribution of 133 chondrite radiants.

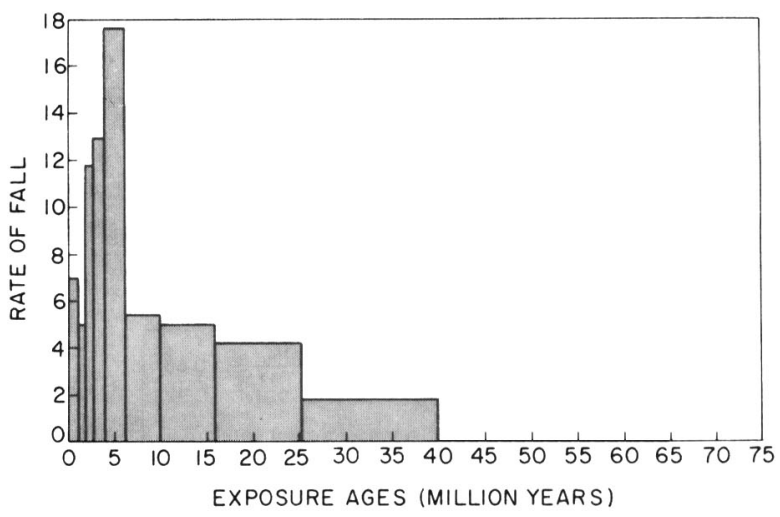

Figure 3.-Observed distribution of chondrite exposure ages.

the Mars-crossing asteroids, typical ring asteroids, Hilda and Trojan asteroids, various short-period comets, and long-period comets. The evolution of these orbits was calculated by use of a revised version of the Monte Carlo method developed by Arnold $(1965 a, b)$, and the distribution of the observable quantities calculated from those orbits terminating with Earth impact. Comparison of the calculated and observed data showed that none of these sources yielded data that agreed with the observed data. In fact, it was shown that only an initial Earth-crossing orbit with perihelion near Earth, aphelion near Jupiter (i.e., $\sim 4.5 \mathrm{AU}$ ), and low inclination would be satisfactory. Calculated exposure ages for such a source are shown in figure 5 . The difficulty 


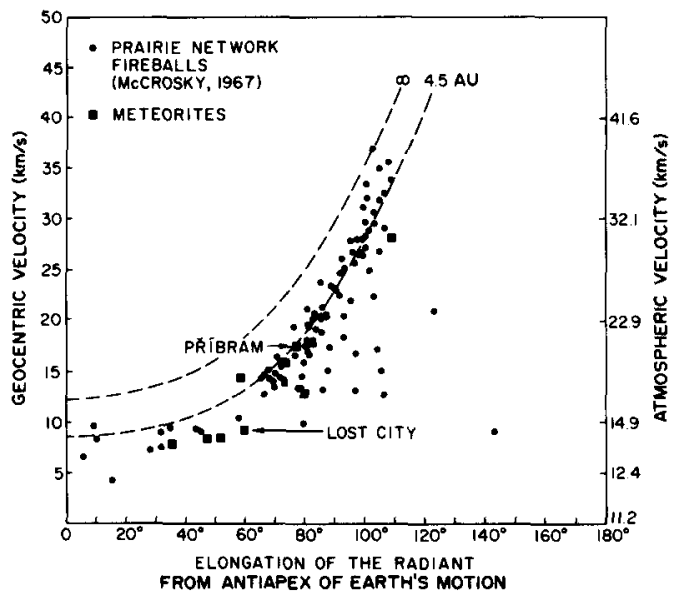

Figure 4.-Observed distribution of geocentric velocities and radiants for Prairie Network fireballs (circles) and the better determined meteorite orbits (squares). The curve marked $\infty$ is the boundary between elliptic and hyperbolic orbits; the other curve is the locus of relatively low-inclination orbits with aphelia at $4.5 \mathrm{AU}$.

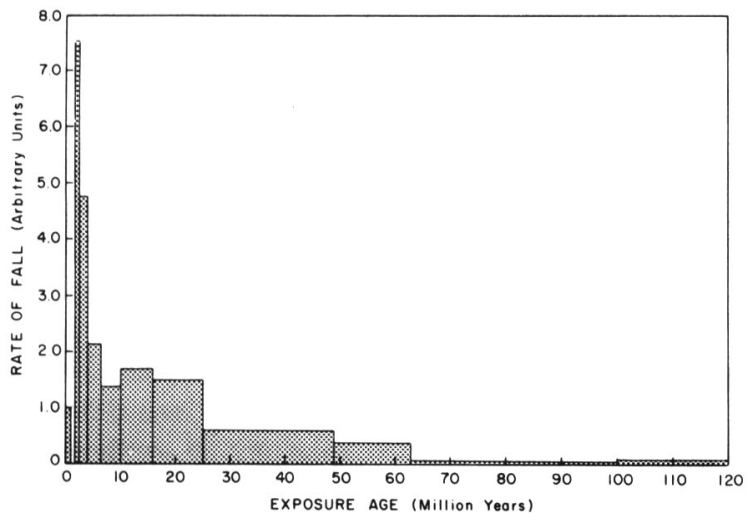

Figure 5.-Calculated distribution of exposure ages for a starting orbit resulting in Earth impacts corresponding to the low-velocity component of the Prairie Network flux. Aphelion $=4.50 \mathrm{AU}$, perihelion $=1.01 \mathrm{AU}$, inclination $=2^{\circ}$. This dynamically determined distribution is very similar to that observed for chondrites, except for those few with exposure ages greater than 50 million yr. These will probably be removed by collisional destruction.

with this orbit is that no family of bodies with such orbits is known. It was suggested (Wetherill, 1969) that a more plausible model would be one in which the observed data were augmented by a component of higher velocity bodies that fail to survive passage through Earth's atmosphere. 


\section{RECENT DEVELOPMENTS}

Work done on this problem during the last $2 \mathrm{yr}$ has confirmed and extended these earlier conclusions. In particular, no satisfactory way has been found for removing from the principal belt of asteroids a significant quantity of relatively unshocked material on the necessary time scale. Other asteroidal sources continue to appear unsatisfactory. The principal new developments during the last $2 \mathrm{yr}$ are the recognition of the fact that the Prairie Network fireball data agree very well with the results predicted for short-period comets of Jupiter's family (and not for other possible sources) and the experimental work of Gault (1969) showing that finite-sized bodies can be broken into fragments much more readily than semi-infinite targets.

It now appears very likely that the Prairie Network fireballs are derived from short-period comets or possibly from related bodies having the same orbital history but less visible as a consequence of containing a smaller fraction of volatile matter. In any case, the identification of the fireballs with these comets shows that objects hundreds of kilograms in mass are associated with these bodies, only a small fraction of which mass can be volatile matter. Consequently, in at least this sense there must be "dead comets," as discussed by Öpik (1963).

\section{COMETS AS SOURCES OF FIREBALLS AND CHONDRITES}

In figure 4, the Prairie Network results are plotted on a diagram where the ordinate is the geocentric velocity and the abscissa is the elongation of the geocentric radiant (corrected for zenith attraction). The scale on the ordinate at the left is the geocentric velocity prior to acceleration by Earth's gravitational field; that at the right is the actual velocity at which the body enters the atmosphere with its velocity augmented by Earth's gravitational field. Values of the elongation of the radiant near $0^{\circ}$ correspond to objects of low inclination that are near their perihelion, when they are moving more rapidly than Earth and are overtaking Earth. Values near $180^{\circ}$ correspond to the opposite situation: bodies near their aphelion; i.e., with orbits within that of Earth. The curve marked $\infty$ is the boundary between elliptic heliocentric orbits and hyperbolic orbits not bound to the solar system. The other curve bounds the regions for which objects of low inclination (i.e., $\leqslant 15^{\circ}$ ) have their aphelia greater or less than $4.5 \mathrm{AU}$. Orbits plotted to the right of this curve have aphelia less than $4.5 \mathrm{AU}$ and have escaped Jupiter's "sphere of influence" but nevertheless are still subject to strong perturbations by Jupiter. The exact position of this boundary is slightly dependent on the inclination, but for the values of the inclination actually observed, this is not significant.

The Prairie Network fireball points are seen to be displaced along the 4.5 AU curve over a wide range of geocentric velocities. This is characteristic of bodies whose orbital evolutions have been primarily determined by proximity to Jupiter. In contrast to this, a body crossing only Earth's orbit will tend to 
preserve a constant geocentric velocity as a consequence of the approximate conservation of its kinetic energy in geocentric coordinates at the point of close approach to Earth. Such a body will evolve horizontally on a diagram of this kind, resulting in frequent high values of the elongation of the radiant. On the other hand, the Jupiter perturbations tend to conserve the same quantity in Jupiter's frame of reference, resulting in a wide spread of geocentric velocities, as exhibited by the Prairie Network fireballs. This is the essential reason why predicted data for bodies with initial Earth-crossing orbits well inside Jupiter's orbit, such as most asteroidal sources, fail to agree with the Prairie Network data.

There are several possible meteorite sources whose orbital evolution is dominated by Jupiter. These are the short-period comets of Jupiter's family and collision ejecta from the Hilda $(a \sim 4.0 \mathrm{AU})$ and Trojan $(a \sim 5.0 \mathrm{AU})$ families of asteroids. Ejecta from the latter two sources that are not stabilized by the commensurability stabilizing the asteroid orbits themselves will be strongly perturbed by Jupiter but will seldom achieve perihelia within that of Earth. The low Jovicentric velocity of these bodies may lead to Jupiter capture; more probably, interaction with the eccentric components of Jupiter's velocity will accelerate the object into Saturn crossing, and ultimately to ejection from the solar system.

The orbits of the short-period comets will evolve in a somewhat different way. If their perihelia are initially not too distant from Earth's orbit (i.e., 1 to $2 \mathrm{AU}$ ), Jupiter perturbations acting near their aphelia will frequently move the perihelia just inside Earth's orbit. Following this, close approaches to Earth will occasionally move the aphelia within 4.5 AU. Escape from Jupiter's sphere of influence may also be aided by nongravitational accelerations, as discussed by Marsden (1968). Although most cometary orbits will suffer the fate of ejection from the solar system (as was the case for the Hilda and Trojan asteroidal ejecta), a small but significant fraction of short-period comet orbits will evolve so that their aphelia are $\sim 4.5 \mathrm{AU}$ and their perihelia $\sim 1 \mathrm{AU}$. From such orbits, meteoroids with the orbital characteristics of the Prairie Network fireballs are derived. If, as proposed by Öpik (1963), there is a residual nonvolatile portion of the comet remaining after the volatile gases, which cause the comet to be visible, have evaporated after $\sim 1000 \mathrm{yr}$, this nonvolatile component will comprise a "dead comet" that will still have, in many cases, a dynamical lifetime of $10^{6}$ to $10^{7} \mathrm{yr}$. This model fits the observed dynamical and physical properties of the Prairie Network fireballs, and no other source has proven to be satisfactory. Predicted data calculated by the Monte Carlo method for residua from comet Neujmin 2 are shown in figure 6, and are seen to resemble closely the distribution found observationally for the fireballs (fig. 4). Similar results are found for any orbit with aphelion within Jupiter's orbit and perihelion less than about $2.0 \mathrm{AU}$. About 10 percent of the observed short-period comets fulfill these criteria; the remainder will give similar points but with greatly reduced mass yields. The results of similar Monte Carlo 


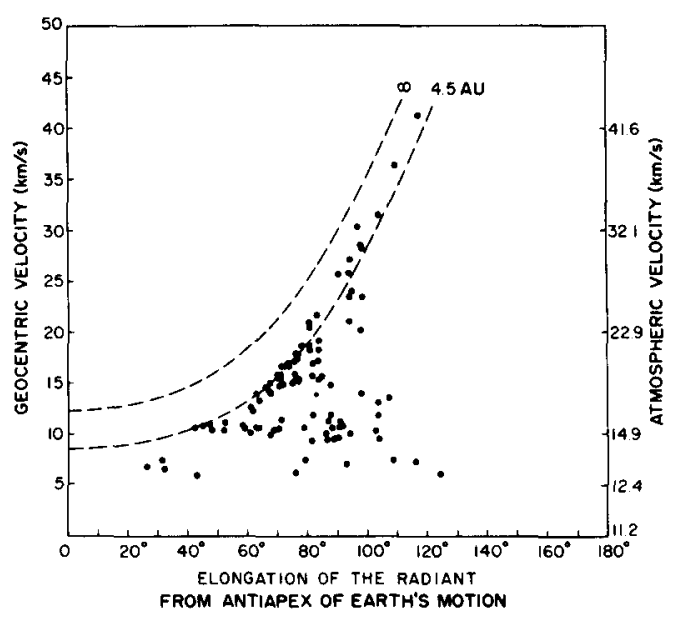

Figure 6.-Calculated distribution of geocentric velocities and radiants for Earth impacts resulting from a starting orbit equivalent to the observed short-period comet Neujmin 2. Aphelion $=4.79 \mathrm{AU}$; perihelion $=1.32 \mathrm{AU}$; inclination $=10.6^{\circ}$; and Earth impact efficiency $=0.24$ percent. Earth impacts requiring more than 30 million yr have been removed because these bodies would probably be destroyed by collisions in the asteroid belt. Inclusion of these events would not affect the distribution very much.

calculations for several other comets are being published elsewhere (Wetherill, 1971).

From this work it is believed that not only are most of the smaller meteors of cometary origin, as has been known for a long time, but also the massive objects observed by the Prairie Network. Consequently, essentially all of the extraterrestrial flux on Earth is derived from comets. There remains the question of whether the meteorites and the chondrites in particular can be identified with this source. It would not be expected that objects with initial atmospheric velocities greater than about $20 \mathrm{~km} / \mathrm{s}$ would survive passage through the atmosphere unless they are unusually large. Therefore it may be anticipated that only the lower velocity component of the data shown in figure 4 would be represented in the material reaching Earth. From figure 4 it may be noted that almost all of these low-velocity bodies have elongations of the radiant less than $90^{\circ}$. This is in accord with the observational data for chondrites (fig. 2) and also leads to the correct distribution of fall times (fig. 1).

The calculated exposure ages would also be similar to those of figure 5. The interpretation of the exposure age for a cometary source depends on the comet model employed. If it is thought that chondrites are buried within the volatile matter of the comet and become separated following the loss of the volatile matter, then the exposure age would start immediately after this loss. As mentioned above, the time $(\sim 1000 \mathrm{yr})$ required for loss of the volatile matter 
is small compared to the exposure age and can therefore be neglected. On the other hand, it could also be that the cometary core is a solid piece of chondritic material hundreds of meters in dimension, the interior of which will be initially shielded from cosmic rays. This model then is in many ways equivalent to an Apollo asteroid source in that the meteorites are derived from a dense, nonvolatile body in an Earth-crossing orbit. Unlike the observed Apollo asteroids, these bodies will predominantly have aphelia near $4.5 \mathrm{AU}$. As discussed elsewhere (Wetherill and Williams, 1968), it is quite likely that Apollo asteroids with large aphelia have escaped detection, and as Öpik (1963) has argued, it is also probable that even the observed Apollo asteroids are cometary cores. If these statements are accepted, therefore, this model of the cometary source becomes identical to an Apollo asteroid model for the origin of chondrites and would also be acceptable as the source of Prairie Network fireballs.

There is evidence that at least the present flux of chondrites is derived from a small number of sources. The most compelling evidence of this kind is that a large number of hypersthene chondrites appear to have experienced a common shock impact within the last 500 million yr (Heymann, 1967). As discussed elsewhere (Gopalan and Wetherill, 1971), this event is not well dated and could have occurred during the last 50 million $\mathrm{yr}$. Therefore, these data are not in themselves strong evidence against a cometary origin of chondrites, but do support the second alternative discussed above; i.e., derivation of chondrites from large comet cores rather than from many small pieces.

In summary, when one includes the effect of the atmosphere as a velocity filter, it turns out that short-period comets satisfy the dynamical requirements for chondrites as well as for fireballs.

Probably the principal difficulty in identifying the chondrites with the fireballs is that the typical fireball apparently has a density lower than that of chondrites and tends to disintegrate in the atmosphere more readily than expected for chondrites. The Lost City meteorite had an aerodynamically determined density higher than that of a typical fireball; on the other hand, Príbram was a typical fireball, with a calculated density, if anything, lower than average. Some evidence for associating typical fireballs with at least one class of chondrites is provided by Revelstoke, a type 1 carbonaceous chondrite recovered following the disintegration of a very large fireball corresponding to an incident mass of hundreds of megagrams, most of which failed to penetrate the atmosphere. The question of the association of more dense stones with the more friable material of a typical fireball remains open.

The identification of possible sources for the highly differentiated meteorites, the achondrites and irons, is more difficult because of the paucity of dynamical data available for these bodies. The high exposure ages of iron meteorites are probably indicative of an asteroidal origin, although a completely satisfactory theory of their mode of derivation from the asteroid belt remains to be developed. 


\section{COLLISIONAL DESTRUCTION}

The other major development in the last few years has been the experimental work of Gault (1969) on the fragmentation of finite-sized targets.

It has long been recognized that meteoritic bodies will undergo collisions with asteroidal debris and cometary meteors and will thereby be reduced in mass. Earlier calculations (Wetherill, 1967) showed that "space erosion" by micrometeorite bombardment was probably of minor importance. However, these same calculations showed that total destruction by a single impact of the meteorite in space might be sufficiently probable to play a minor role in limiting the observed exposure ages of chondrites to a few tens of millions of years. This higher probability for total destruction is in large measure a consequence of the fact that fragmentation of a finite-sized body can result from hypervelocity collision with much smaller masses. Cratering experiments indicate that collisions in the asteroid belt between small and large bodies should produce craters on the larger body, the mass ejected from the crater into space being about 100 times the mass of the projectile. However, for somewhat larger projectiles, additional damage to the target results from shock waves traversing the body and reflecting from the bounding surfaces. In the earlier calculations (Wetherill, 1967) it was estimated that this effect might increase the ratio of ejected mass to projectile mass to about $10^{4}$ for the case when this additional damage was just sufficient to fragment the target into a number of pieces.

The experiments of Gault have now shown that ratios of ejected to projectile mass as high as $10^{6}$ are possible. The effect of this new result on cosmic-ray-exposure ages has been evaluated by computing the probability of destruction of bodies in various orbits by collision with a population of objects with orbits distributed similarly to the observed asteroids and periodic comets. Several assumptions were made regarding the total mass and population index of the colliding asteroidal and cometary bodies, based on meteor observations and theoretical studies of fragmentation in the asteroid belt (Dohnanyi, 1970). The effect of the relative velocity of the two colliding bodies was taken into consideration not only insofar as it affects the probability of collision, but also in accordance with its effect on the strength of the collision by use of Gault's experimental result that kinetic energies of $10^{2}$ to $10^{3} \mathrm{~J} / \mathrm{kg}\left(10^{6}\right.$ to $\left.10^{7} \mathrm{ergs} / \mathrm{g}\right)$ will suffice to completely fragment finite-sized bodies.

The results of these calculations are that total destruction by asteroidal fragments and cometary meteors are of comparable importance and that either may predominate, depending on the exact assumptions made regarding the flux of the colliding bodies. For fragmentation energies of $10^{2} \mathrm{~J} / \mathrm{kg}\left(10^{6} \mathrm{ergs} / \mathrm{g}\right)$, a body $50 \mathrm{~cm}$ in radius will have a mean lifetime of about 10 million $y r$. This result is not very sensitive to the orbit assumed for the body. Uncertainties in the flux could easily cause the quantity to be in error by a factor of 10 . Fragmentation energies of $10^{3} \mathrm{~J} / \mathrm{kg}\left(10^{7} \mathrm{erg} / \mathrm{g}\right)$ will increase the lifetime to 
about 100 million yr. In addition, it is possible that meteorite lifetimes may be limited by rotational bursting (Paddack, 1969).

The consequence of these results is that it now seems likely that total destruction by collision will prevent meteorites from having very large cosmic-ray-exposure ages. For the calculations based on the cometary source, this is a secondary effect. In this case, the cosmic-ray-exposure ages will be primarily controlled dynamically; the probability of these objects surviving ejection from the solar system by Jupiter perturbations for more than $\sim 25$ million yr is not large anyway. The effect of collisional destruction will be to cut off the high exposure age "tail" on figure 5 and bring the calculated results into even better agreement with the observed data.

For asteroidal sources, the effect is greater because for collision lifetimes as short as $10^{7} \mathrm{yr}$ it is no longer possible to obtain the long exposure ages calculated for meteorites derived from Mars-crossing asteroids and, to a lesser extent, from Earth-crossing Apollo asteroids. This does not, however, increase the plausibility of deriving asteroids from these sources. Fragments of objects moving in orbits similar to the observed Mars-crossing or "Mars-grazing" asteroids will have their initial perihelia barely within Mars' aphelion. Multiple perturbations involving elapsed times of the order of $10^{9} \mathrm{yr}$ will be required to perturb this initial orbit into an Earth-crossing orbit. By this time, collisional destruction will have eliminated all of the fragments. If the initial distribution of Mars-crossing orbits were a random one, about 1 percent of the fragments would be perturbed into Earth-crossing orbits sufficiently rapidly to avoid destruction. However, as pointed out above, the distribution of initial orbits is a very special one, and a distinct delay on the order of $10^{9} \mathrm{yr}$ is involved prior to appearance of the fragments in Earth-crossing orbits.

This difficulty of survival can be avoided by theories in which chondrites are derived from Apollo asteroids as a result of partial or complete fragmentation of the asteroid as it passes through the asteroid belt. In this case, the fragments are produced directly in Earth-crossing orbits and no delay of the type discussed above occurs. As discussed earlier in this paper, the most plausible cometary model is a theory of this kind in which the "Apollo asteroids" are cometary cores with aphelia near 4.5 AU. Alternative Apollo asteroid theories in which they are derived from ring asteroids or from Mars-crossing asteroids are less satisfactory. A short lifetime for collisional destruction removes the objection to theories of this kind raised previously (Wetherill and Williams, 1968) that predicted exposure ages are far too long. At the same time, this makes it more difficult to reconcile the other observations with the results predicted for such a model.

The large observed excess of radiants less than $90^{\circ}$ requires that most Earth impacts occur while the meteorites are near their perihelion. This fact in turn requires that a large fraction of the meteorites be produced immediately after the Apollo asteroid is perturbed into Earth crossing. With the passage of time, the Apollo asteroid will be perturbed by Earth and Venus into orbits for which 
its aphelion is near Earth as frequently as its perihelion. This tends to produce a symmetric distribution of radiants for low-velocity bodies, and an excess of radiants greater than $90^{\circ}$ for higher velocity bodies. This has always been a problem with theories of this kind. Short collisional lifetimes aggravate this difficulty by relatively deemphasizing fragments produced when the Apollo asteroid first becomes Earth crossing in favor of those produced later after the perihelion of the source has become randomized.

\section{SUMMARY}

These calculations indicate excellent agreement between observed and predicted orbits of Prairie Network fireballs, if it is assumed that fireballs are derived from remnants of short-period comets of Jupiter's family. No such satisfactory agreement has been found for any other proposed source. The distribution of radiants and time of fall observed for chondrites will also be reproduced by this source, provided that consideration is given to the fact that Earth's atmosphere will permit low-velocity bodies to survive but will destroy high-velocity bodies. Again, no other proposed source has been found to be adequate.

It now appears likely that the mean lifetime of chondrites is limited to $\sim 10^{7} \mathrm{yr}$ by the high probability of complete fragmentation following impact by smaller bodies. This improves the agreement between the observed cosmic-ray-exposure ages and those predicted for a cometary source. This also requires some modification of the earlier discussions of alternative sources but does not result in their becoming more satisfactory.

Note added in proof: Recent work by P. Zimmerman and the author shows that it is possible to inject fragments of the size of small asteroids into the 2:1 Kirkwood gap. Although the resulting libration will enable the body to avoid Jupiter, a meteorite-sized fragment ejected at a velocity of about $200 \mathrm{~m} / \mathrm{s}$ as a result of a collision can escape the libration region and be in an orbit similar to those of the short-period comets, as discussed in this paper. This mechanism has not yet been sufficiently quantitatively evaluated in order to learn its importance as a source of meteorites.

\section{REFERENCES}

Arnold, J. R. 1965a, The Origin of Meteorites as Small Bodies, 2, The Model. Astrophys. J. 141, 1536-1547.

Arnold, J. R. 1965b, The Origin of Meteorites as Small Bodies, 3, General Considerations. Astrophys. J. 141, 1548-1556.

Dohnanyi, J. S. 1970, On the Origin and Distribution of Meteoroids. J. Geophys. Res. 75 , 3468-3493.

Gault, D. E. 1969, On Cosmic Ray Exposure Ages of Stone Meteorites (abstract). Meteoritics 4, 177.

Gopalan, K., and Wetherill, G. W. 1971, Rubidium-Strontium Studies on Black Hypersthene Chondrites: Effects of Shock and Reheating. J. Geophys. Res., in press.

Heymann, D. 1967, On the Origin of Hypersthene Chondrites: Ages and Shock Effects of Black Chondrites. Icarus 6, 189-221.

McCrosky, R. E. 1967, Orbits of Photographic Meteors. Smithson. Observ. Special Rept. 252. 
Marsden, B. G. 1968, Comets and Non-gravitational Forces. Astron. J. 73, 367-379.

Öpik, E. J. 1951, Collision Probabilities With the Planets and the Distribution of Interplanetary Matter. Proc. Roy. Irish Acad. Sect. A 54, 164-199.

Öpik, E. J. 1963, Survival of Comet Nuclei and the Asteroids. Adv. Astron. Astrophys. 2, 219-262.

Öpik, E. J. 1966, The Stray Bodies in the Solar System, 2, The Cometary Origin of Meteorites. Adv. Astron. Astrophys. 4, 302-336.

Paddack, S. J. 1969, Rotational Bursting of Small Celestial Bodies: Effects of Radiation Pressure. J. Geophys. Res. 74, 4379-4381.

Wetherill, G. W. 1967, Collisions in the Asteroid Belt. J. Gcophys. Res. 72, 2429-2444.

Wetherill, G. W. 1968a, Time of Fall and Origin of Stone Mcteorites. Science 159, 79-82.

Wetherill, G. W. 1968b, Dynamical Studies of Asteroidal and Cometary Orbits and Their Relation to the Origin of Meteorites. Origin and Distribution of the Elements (ed., L. Ahrens), pp. 423-443. Pergamon Press. New York.

Wetherill, G. W. 1969, Relationships Between Orbits and Sources of Chondritic Meteorites. Meteorite Research (ed., P. M. Millman), pp. 573-589. D. Reidel. Dordrecht.

Wetherill, G. W. 1971, Origin and Age of Chondritic Meteorites. Vinogradov 75th Anniv. Vol. Moscow. In press.

Wetherill. G. W., and Williams, J. G. 1968, Evaluation of the Apollo Asteroids as Sources of Stone Meteorites. J. Geophys. Res. 73, 635-648.

\section{DISCUSSION}

KESSLER: I have always been leary of comparing observational data from two different sources. For example, the probability of observing a fireball of a given mass may vary as something like velocity to the third or fourth power, whereas the probability of observing an asteroid or comet in space may be inversely proportional to velocity. The results would be to reduce the relative number of high-velocity fireballs and perhaps increase the number of high-velocity asteroids or comets. I am wondering if you considered these selection effects; and, if so, what effects they would have on your conclusions.

WETHERILL: There are only a few comets known with aphelia between 4.5 and $5 \mathrm{AU}$. However, whether there are more or less is minor in importance. Any of these will evolve in such a way as to give a similar distribution on the velocity-elongation diagram. Whether there are strong biases in the Prairie Network can best be answered by McCrosky.

MCCROSKY: The bias is in favor of observing higher velocity objects.

WHIPPLE: I question the large radii obtained by Wood and Goldstein. How can one have very much confidence in the radiation loss on a body which we know so little about? The outer surfaces of asteroids could have very low thermal conductivity and prevent heat loss. Consequently the interior could have been much hotter and the bodies would have been considerably smaller. It is very hard to know what value to put in.

ANDERS: Fricker considered a thin surface layer but it was not significant. If you make it thick enough it will be significant.

ARRHENIUS: There is another way to approach this discrepancy; that is, by the rather large uncertainties in the cooling rates. The diffusion coefficients that are used are extrapolated from higher temperatures, and there are rather large uncertainties. Another one is based on the fact that minor components such as phosphorus and hydrogen will increase the diffusion rate. All this would work in the direction of making the size of the body smaller.

WETHERILL: I would like to say something about this question of large bodies. I do not see any compelling reason for not believing that asteroidal and cometary masses are distributed in a similar way. Most of the asteroidal mass is in a few large bodies, and hence these few large bodies might be expected to contribute most asteroidal fragments. In the 
same way, most of the cometary fragments could be derived from a few large comets rather than a large number of small ones.

UREY: It seems to me that the rather large objects must have been present in the primitive solar nebula and that they collided with the planets during their accumulation. In this way the tilt of the axes of the planets from the vertical to the ecliptic plane can be accounted for; and, in fact, the reverse rotation of Venus and the tilt of the axes of Earth, Mars, Uranus, and others with the exception of Jupiter must have originated in this way. 1 have suggested that many lunar-sized objects were present. It has been discussed by Marcus, Safranov, and, more recently, by Singer. The particular case of Uranus was called to my attention by Gold quite some years ago.

KENKNIGHT (submitted after meeting): Although comets might be attractive for meteors and some chondrites, the chemistry of the achondrite meteorites strongly suggests origin on, or in association with, a large enough body to have been strongly heated at origin. The chemistry and mineralogy of achondrites suggest a magmatic relation to material of the chondrite type. The structure and composition of the brecciated achondrites suggest histories as complicated as lunar surface breccias, including magmatic differentiation, brecciation or surface effusion, recrystallization, and further brecciation (Duke and Silver, 1967). Wasson and Wai (1970) give 11 reasons for believing the enstatite chondrites and enstatite achondrites form a systematic sequence driven by a heat source external to the parent body that was increasing with time and sufficiently intense to cause partial melting of silicates. The occurrence of gas-rich achondrites and the specific pattern of enrichment in $\mathrm{C}, \mathrm{Ni}, \mathrm{Br}$, and $\mathrm{Bi}$ in addition to the noble gases in these achondrites suggests (Mazor and Anders, 1967; Muiller and Zahringer, 1966) that carbonaceous chondrite material was added as an impurity at a parent body surface and then incorporated in a breccia during impact. These conditions at or near a meteorite parent body surface are consistent with the identification of the surface reflectivity of Vesta in the earlier paper by Chapman, Johnson, and McCord ${ }^{1}$ with a eucritic achondrite, Nuevo Laredo.

WETHERILL: KenKnight's statement covers a very wide amount of territory in a very few sentences, some of which I agree with, some of which I do not. Therefore, I will confine my remarks to his first sentence, which may be taken to represent a summary of the remainder. I agree that the chemistry of the achondritic meteorites strongly suggests origin in a large enough body to have been strongly heated at the time of its formation. The same is true of the metamorphosed chondrites for that matter. On the other hand, we do not know if asteroids were strongly heated at the time of their formation, nor do we know that the cores of the comets were not. We do know that this heating took place during the formation interval of the solar system; and, therefore, an understanding of the very complex processes that took place in this interval is necessary to discuss these questions in a meaningful way. 1 am not convinced that any of us know that much about it, even though some people purport to.

\section{DISCUSSION REFERENCES}

Duke, M. B., and Silver, L. T. 1967, Petrology of Eucrites, Howardites, and Mesosiderites. Geochim. Cosmochim. Acta 31, 1637-1665.

Mazor, E., and Anders, E. 1967, Primordial Gases in the Jodzic Howardite and the Origin of Gas-Rich Meteorites. Geochim. Cosmochim. Acta 31, 1441-1456.

Müller, O., and Zahringer, J. 1966, Chemische Unterschiede bei Uredelgashaltigen Steinmeteoriten. Earth Planet. Sci. Lett. 1, 25-29.

Wasson, J. T.. and Wai, C. M. 1970, Composition of the Metal, Schreibersite, and Perryite of Enstatite Achondrites and the Origin of Enstatite Chondrites and Achondrites. Geochim. Cosmochim. Acta 34, 169-184.

\footnotetext{
${ }^{1}$ See p. 51 .
} 